\title{
Chapter 1 \\ Health Information Technology \\ as Premise for Data Science in Global \\ Health: A Discussion of Opportunities and Challenges
}

\section{Louis Agha-Mir-Salim and Raymond Francis Sarmiento}

\begin{abstract}
Background Healthcare systems function as an important component and a contributing factor in global health. The application of information technology (IT) in healthcare systems function as a basis for the utilization of data science, whichin its practical application-not only provides opportunities to increase the quality of care, improve efficiency, and decrease costs but also buries the risk of hindering existing workflows, decreasing staff satisfaction, and further siloing access to patient data. Methods Three different applications of health information technology (HIT), applied in the context of data science, will be examined in this chapter with regard to their opportunities and challenges for the system and, as a result of this, for global health. Results Electronic health records, health information exchange, and artificial intelligence have great potential to alleviate some of healthcare systems' greatest burdens and make modern medicine more evidence-based, yet their successful implementation yields a multidisciplinary approach, constant development and evaluation, and collaboration amongst all stakeholders. Conclusions Stakeholders and implementers must consider the opportunities and challenges that come with the planning, implementation, and maintenance of HIT in order to minimize negative impacts and leverage its full potential for an overall improvement of global health.
\end{abstract}

Keywords Health information technology $\cdot$ Electronic health records $\cdot$ Health information exchange $\cdot$ Artificial intelligence (AI)

\section{Learning Objectives}

In this chapter, we discuss the role of health information technology (HIT) in increasingly complex, challenging, and constantly evolving healthcare systems with regard to its role in data science (for more details on the methods of data science, please refer

\footnotetext{
L. Agha-Mir-Salim ( $ه)$

Faculty of Medicine, University of Southampton, Southampton, UK

e-mail: mirsalim@mit.edu

Laboratory for Computational Physiology, Massachusetts Institute of Technology, Cambridge, MA, USA

R. F. Sarmiento

University of the Philippines Manila, Manila, Philippines
} 
to subsequent chapters of this book). We focus on how modern technologies, such as electronic health records, health information exchange, and artificial intelligence, find application in healthcare systems and how this, in turn, provides opportunities to leverage data for improvement of care quality and efficiency. We also approach the difficulties and unintended consequences that arise from the adoption and integration of such technologies into existing healthcare systems and their workflows. Besides considering technical barriers, we also examine the human layer as an important component of driving change within this domain. Finally, we will draw conclusions on where HIT is adding value for purposes of data science and provide recommendations where barriers yet need to be overcome in maximizing their potential to enhance healthcare as a highly complex component of global health.

\subsection{Background}

Although not explicitly mentioned in definitions of global health, healthcare systems can be viewed as an instrument or executive tool that governments and organizations deploy in striving for global health. With one of its core aspects comprising "both [the] prevention in populations and clinical care of individuals" (Koplan et al. 2009), global health is very much is reliant on healthcare systems as a subordinate framework to achieve its set goals.

Nevertheless, healthcare in itself is a highly complex and information-intensive field. The increasing integration of technology has allowed healthcare decisionmakers to collect, process, and analyze this data for a more effective care delivery while enhancing the safety of patients and consumers (Singh and Sittig 2016). Considering the newly gained abundance of all health data - collected through health information technology (HIT) — coupled with the drastic developments in the field of data science (for more details on the methods of data science, please refer to subsequent chapters of this book) over the last decade, allows us to reimagine the practice of healthcare with evermore applications to address the industry's dynamic challenges of improving care quality, decreasing costs, guaranteeing equitable access, and fostering public health surveillance; all key components of achieving Universal Health Coverage (World Health Organization 2013).

In efforts to improve healthcare through digitization, varying approaches within and between countries have been taken, including the introduction of electronic health records (EHRs), telehealth and telemedicine, electronic learning and decision support systems, mobile health (mHealth) applications, real-time surveillance systems, artificial intelligence, etc. (Figure 1.1) (World Health Organization 2016). eHealth, often used interchangeably with HIT, is defined as the "the cost-effective and secure use of information and communications technologies (ICT) in support of health and health-related fields, including health-care services, health surveillance, health literature, and health education, knowledge and research" (World Health Assembly 2005, p. 121). It facilitates countries' health agenda by improving operational processes of service delivery, building workforce capacity, and engaging all 


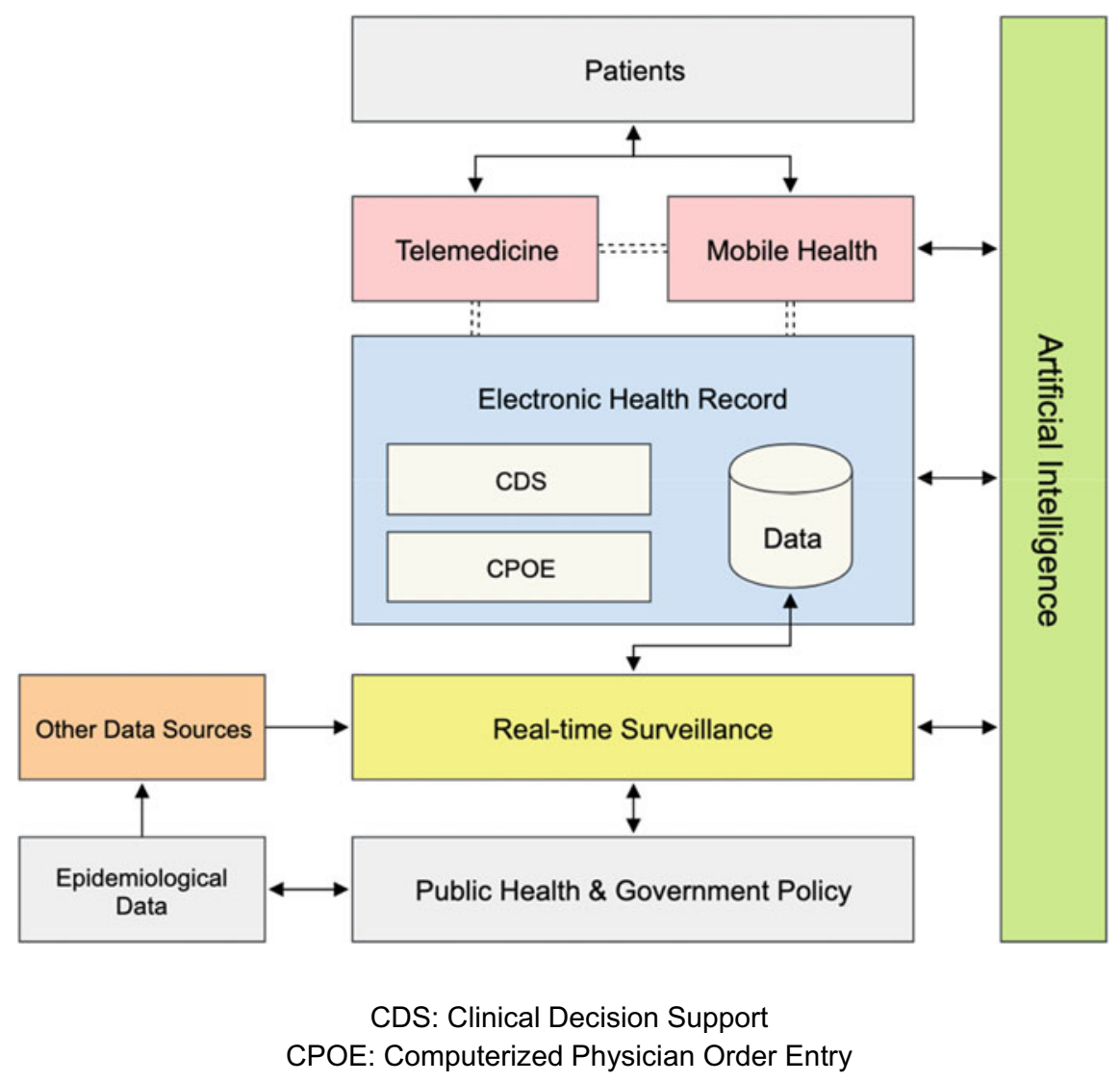

Fig. 1.1 Elements of healthcare information technology

stakeholders in the care process - from the healthcare leaders and managers over healthcare professionals to patients and communities (Al-Shorbaji 2018).

However, uptake of these technologies has been uneven within and across countries due to differing levels of maturity in ICT infrastructure, lack of skilled healthcare personnel, and more. These are often among the fundamental hurdles in updating and maintaining up-to-date HIT, especially in low and middle-income countries (LMICs) (Clifford 2016). In several countries, however, various pilot implementations have been initiated but few have gone to scale (Sundin et al. 2016). This was due to several factors with socio-cultural challenges at the core despite great financial, clinical, and logistical enablement from supporting organizations and responsible governments (Clifford 2016). Additionally, countries, particularly LMICs, continue to be challenged by the rapid development of HIT interventions, such as constantly evolving EHR infrastructures. Successful adoption and implementation of HIT solutions are also constrained by other challenges such as poor ICT infrastructure and access, misalignment between HIT investments and the national health agenda, and poorly 
defined enterprise architectures and standards (Baller et al. 2016). All of these factors limit the accountability, scalability, sustainability, and resilience of health systems. Of course, the role of data science as a layer of added value, enabled through HIT, is limited in settings where HIT integration is low. On the other hand, the impact of data science in LMICs is likely to be more far-reaching with the integration of simple HIT applications, acting as low-hanging fruits to improve care (Costa et al. 2012). Yet it is imperative to remain realistic and consider the structural, social, and financial obstacles we are faced with.

In the following section of this chapter, we will closely examine three frequently applied examples of HIT and how these play a role in data science. The technologies we will focus on are EHRs, health information exchange (HIE), and artificial intelligence (AI).

Building the basis for the application of data science techniques by enabling the availability of vasts amount of data, EHRs display a means for the collection and storage of data. Leading on from here, HIE allows for the exchange of this data, and AI algorithms will subsequently grant the analysis and provision of predictive models to be applied in clinical practice and administration. Hence, these three technologies portray different aspects of the journey a patient's clinical data takes-from storage, over exchange, to analysis. Additionally, they symbolize examples of relevant HIT advancements over the years in a chronological manner.

\subsection{Examples of HIT and Their Application in Data Science}

\subsubsection{Applied Example No. 1: Electronic Health Records (EHRs)}

One of the key prerequisites for improving the delivery of care services through data science is the efficient collection, storage, analysis, and exchange of health information across different service levels in a secure yet practical fashion. Data science tools, such as machine learning and deep learning, rely heavily on massive collections of labelled structured and unstructured data, in order to train models and subsequently improve them to guide decision. Hence, a data acquisition pipeline is paramount. For this purpose, EHRs have become indispensable tools to carry patient health information and facilitate its use between different levels of care. This is reflected by the increasing number of national HIT strategies around the globe, starring the implementation and development of EHR systems (World Health Organization 2016).

High income countries have seen the largest investments in EHR systems. In the United States, the Health Information Technology for Economic and Clinical Health Act (HITECH Act of 2009) spurred on the rapid digitization of the healthcare delivery system, eventually culminating in the Medicare and Medicaid EHR Incentive Programs (Washington et al. 2017). Here, EHRs have provided accurate 
and up-to-date information at the point of care, enabled quicker access to patient records for more coordinated care among healthcare providers, and reduced healthcare costs by decreasing the amount of paperwork and duplicate diagnostic exams while streamlining coding and billing services as a result of complete and accurate documentation of all transactions.

However, the adoption and implementation of EHRs have been a great source of both satisfaction and consternation, particularly in the last ten years. In recent years, physicians' satisfaction with EHRs have become universally low (Shanafelt et al. 2016), probably due to an increasing workload and the incentives received as a result of complete documentation. Unintentionally, this has gradually become a burden for providers around the world by negatively affecting their relationship with patients and clinical workflows (Goldberg 2018). In 2016, a study by Shanafelt, et al., revealed that physicians who used EHRs and computerized physician order entry (CPOE) systems, e.g., electronic prescribing, demonstrated a lower level of work satisfaction due to the amount of time spent on clerical tasks and had an increased risk of burnout (Shanafelt et al. 2016). Moreover, physicians have progressively become concerned that medical malpractice liability may increase with the implementation of CPOE systems due to the increased documentation of computer-related errors (Mangalmurti et al. 2010). In LMICs, this set of problems could prove even more troublesome as the working conditions for health professionals are likely to be even more challenging. Adding an EHR system without taking into consideration all its implications could have disastrous consequences for all aspects of care provision and staff satisfaction.

Further examples of this ambivalence are tools that count as subordinate functions of EHRs, including CPOE systems and Clinical Decision Support (CDS) systems. As mentioned above, electronic prescribing is one prominent example of a CPOE system. As opposed to handwritten prescriptions, the electronic prescribing alternative promises greater prevention of medication errors. Reasons for this are increased completeness, standardization, and legibility of pharmaceutical prescriptions, as well as their frequent integration with CDS tools (Puaar and Franklin 2017). CDS systems are digital applications to "provide alerts, reminders, prescribing recommendations, therapeutic guidelines, image interpretation, and diagnostic assistance" (Khairat et al. 2018) and are often deployed to complement CPOE systems. This integration leads to an enhancement of patient safety with lower prescriptions errors and improved interprofessional communication between healthcare providers (Mills et al. 2017).

However, despite the proven potential of CDS for electronic drug alerts by reducing the number of adverse drug events and lowering healthcare costs (Ash et al. 2007); (Weingart et al. 2009), it is one of the leading causes of alert fatigue in healthcare providers. Alert fatigue describes a phenomenon where the user, i.e. the clinician, actively ignores or dismisses pop-up windows, warning the user of possible errors or dangers with clinical information that they entered. Alerts for drug-drug interactions, pre-existing drug allergies, weight-adjusted dosing etc., often appear very frequently, hence 'fatiguing' the user's attention to them (Backman et al. 2017). It has been shown to debilitate the power of alerts, especially if deemed obvious or irrelevant, leading clinicians to dismiss future pop-ups without reading potentially important alert messages (Ash et al. 2007). Consequences of alert fatigue could lead 
to the user's impression of being supervised and treated as distrusted in their own decision-making with resentment due to the continuous interruption in their work. In order to prevent this, it is therefore imperative to ensure user-friendliness, along with the relevance and appropriateness of alerts in the given clinical context when designing CDS systems (Ash et al. 2007).

Considering these benefits and drawbacks of EHRs, along with CPOE and CDS, that are inherent to their integration, they certainly all allow for the further collection of data by the digitization and integration of workflows, such as the prescription of medication. Before the integration of HIT, these processes either used to be analogue or non-existent, whereas now they can be streamlined and interoperable with one another. This may ease the documentation burden of handwritten notes and enable the collection of even more clinical data, which can much more readily find application for research or in public health. However, as with all technological novelties in healthcare, if not integrated well, all systems can become cumbersome and in several ways harmful to deliver good care. Healthcare professionals may circumvent the correct use of these electronic systems, which may negatively impact overall efficiency, effectiveness of care, and patient safety (Blijleven et al. 2017).

The full impact of EHRs on data science in global health is challenged by smaller and larger scale problems, ranging from human issues to technical difficulties. It begins with the above mentioned issues, e.g., low EHR usability and staff resistance, and ends with the major, systems-based problems of healthcare, such as the increase in healthcare cost, the rate of medical errors, or exhaustion and shortage of workforce, all of which limit the integration and adequate maintenance of EHR systems.

\subsubsection{Applied Example No. 2: Health Information Exchange (HIE)}

Health information exchange (HIE) is the mobilization and transfer of electronic health information within and across organizations in a community, region, or country, ideally through interoperable health information systems (Finn 2011). It allows healthcare providers and patients to securely access medical information electronically in order to appropriately and confidentially share patient's health information independent of where they are receiving care (HealthIT.gov 2017). The United States, Canada, Australia, and the UK are some of the countries who have, to a certain extent, successfully implemented regional or state-wide HIEs.

An effective implementation of HIE is critical to provide high-quality, tailored care to patients while reducing costs and increasing access (Sadoughi et al. 2018). To do this, HIE implementation must be aligned with inner-organizational as well as interorganizational needs and priorities, with mutual cooperation and collaboration being crucial in fostering HIE. In 2016, Eden, et al., showed that facilitators and barriers to successful HIE implementation could be categorized as to the completeness of information, organization and workflow, and technology and user needs (Eden et al. 
2016). In particular, the lack of consistent terminology and classification of HIE was found to be a considerable barrier to understanding how an HIE ideally functions, as well as constant changes in sociotechnical systems (Eden et al. 2016). These findings are consistent with the 2016 study of Akhlaq, et al., done for LMICs, wherein they found that successful HIE implementations largely depend on effective policies, strong leadership, and governance in order to create an evidence-based decisionmaking culture within organizations (Akhlaq et al. 2016).

Revisiting the concept of EHRs and thinking a step ahead, being able to not only access local clinical data but to exchange data across departments, organizations, regions, or even nations through HIEs, a whole new extent of data availability becomes apparent. Due to these vast amounts of data being necessary in order to leverage its full potential, it symbolizes a key requirement for data science adding real-world value and effectively integrate AI on a broader scale. Still being far from achieving a smooth and widespread HIE across regions and countries, for the most part, we can only speculate on the impact the analysis of all this data can have once this will be achieved. As a result of these HIE networks, we need to find the value of all the accumulated data, not only by making medical practice more evidence-based but also in the field of, e.g., population-based informatics, or genetic and genomic information. Generally, once data is available, analysing it and drawing conclusions from it for clinical practice is relatively easy as compared to the far greater hurdle of translating these findings from 'bench to bedside'.

Taking another step ahead, blockchain technology (for more details, please refer to subsequent chapters of this book) has been proposed as a tool to provide the necessary features for long sought after advancements in the industry, especially with regard to increased interoperability, high data security, and seamless HIE (Gordon and Catalini 2018). Similarly, a good amount of temperance may also be needed. Blockchain has been envisioned to change the status quo in clinical research, public health, patient identification systems, and self-generated health data (Gordon et al. 2017). It has also been explored in terms of improving global health (Metcalf 2019, p. 415). However, one has to keep in mind that health systems are multifaceted, highly fragmented, and very resistant to change. Thus, expectations should be kept realistic in the face of persistent doubts on its sectoral need, appropriate use, and whether it can truly change existing health systems - particularly on data handling, because of the lack of scalable real-world implementations (Gordon et al. 2017).

Besides the necessary technological prerequisites, key to the successful implementation of an HIE are governance, consistency in technical nomenclature, and effective change management. These three factors are determined to have significant effects on the level of adoption and success that organizations experience when implementing an HIE. As they are extremely difficult to achieve due to the need for many disparate parties to align, conferences can foster the conversations that enable broader and effective change management, bringing all different stakeholders to the table. It outlines the need for collaboration as health data, if collected in an orderly and accessible fashion, is still mostly siloed unless governance and other initiatives drive parties towards unification and liberation of data. 


\subsubsection{Applied Example No. 3: Artificial Intelligence}

Given that EHRs and HIE have done their work to provide data in an accessible and orderly fashion so that it can be further utilized, artificial intelligence (AI) can be applied in helping to improve everyday clinical questions, predict disease outbreaks in LMICs, monitor drug adherence, etc. The application of these new technologies have spurred excitement and brought renewed hope in finding solutions to the intricate and sophisticated problems inherent to global health.

With AI applied to healthcare and global health, we associate the use of computer algorithms and statistical models to enhance the human understanding of complicated medical information and coherences by analyzing medical data. Specifically, AI usually refers to tasks performed by computers that would otherwise require intelligence if executed by humans (The Alan Turing Institute 2018).

The field of AI has evolved over the last 60 years. First described in 1971 in medical publications (Coiera 1996), it is only now that many AI applications have been deployed in healthcare settings and there are signs indicating that AI adoption has been growing exponentially. Areas that would benefit from added value through datadriven solutions can be classified as having either a 'patient focus' and/or 'healthcare provider/payers focus' (Garbuio and Lin 2019). Within clinical medicine, as part of the latter focus, there is a myriad of specialties that would benefit from the integration of AI engines, with possible tasks ranging from natural language processing over clinical decision support to predictive analytics (Dankwa-Mullan et al. 2018); (Yu and Kohane 2018) (for more details, please refer to subsequent chapters of this book). Despite the fact that a range of those AI applications have already proven to perform on par with experienced clinical specialists (Esteva et al. 2017), many experts see AI's future role in complementing human knowledge and decisions, by rapidly exploiting vasts amount of data, instead of replacing doctors (Dankwa-Mullan et al. 2018). Hence, most AI applications in healthcare are aimed at working in synergy with staff instead of striving for a substitution of workforce.

One major application of AI-assisted medicine is the ability to make reliable and accurate predictions on clinical outcomes, hence assisting clinicians in critical everyday decisions, for example by finding the optimal treatment strategy for patients with sepsis (Komorowski et al. 2018) or utilizing warning algorithms and severity of illness scores in intensive care (AIMed 2018). Other examples include radiological or pathological image processing through deep neural networks (Esteva et al. 2017). Hence, machine learning and deep learning, methods of AI, will not only alleviate a great portion of physicians' workload but will also provide more accurate clinical prognoses and enhance diagnostic accuracy (Obermeyer and Emanuel 2016). This triad of features ultimately contributes to ML enhancing patients' outcomes with the adoption of AI in healthcare.

An industry example of an AI application currently in use for global health is IDx, a US-based startup. The company has succeeded in building the first and only FDA authorized AI system for the autonomous detection of retinopathy in adults with diabetes, namely IDx-DR (FDA, 2018). The shift towards AI oriented efforts 
is also being demonstrated by academia, e.g., with the foundation of the Stanford Institute for Human-Centered Artificial Intelligence in early 2019. Other academic examples include free online courses, like MIT's course “Global Health Informatics to Improve Quality of Care” on edX (Celi 2019) or Stanford's Andrew Ng's course in Machine Learning on Coursera, enabling anyone to gain an understanding of health informatics and how to leverage Big Data (Ng 2011).

However, despite all the excitement and the predicted opportunities for bettering healthcare using AI, bringing ML algorithms from a laboratory to the bedside remains a major challenge. Regulatory and ethical issues, such as confirmatory bias or reduced patient safety, have been discussed involving the routine use of AI, with uncertainty regarding the point of sufficient performance of a program and accountability in the event of a medical error (Dankwa-Mullan et al., 2018). The absence of such controlling mechanisms to date raises questions as to whether the use of AI, though it may solve problems and enhance care delivery, may again create new unintended problems, such as reducing efficiency (Yu and Kohane 2018) or questionable clinical success rates of new algorithms, leading to concerns for patient safety.

\subsection{Discussion}

With all of the challenges faced by current global healthcare systems, including an ever-increasing financial burden and an aging population, the use of HIT systems and data science have the potential to improve the effectiveness of treatments while reducing costs and increasing the quality of care, as suggested by many studies (Silow-Carroll et al. 2012); (Murphy 2014); (Sadoughi et al. 2018). Moreover, they may not only enable a more holistic patient care by facilitating collaboration among different service providers but have the potential to improve patients' and providers' experience and satisfaction. Aside from alleviating patients' own burden in receiving appropriate care, the clinicians' experience may also likely be enhanced when care becomes again more efficacious and patient-focused, e.g., when medical records are easily accessible, prescriptions errors would be flagged before having negative effects, or algorithms could give specific treatment recommendations in the management of complex clinical conditions. In an ideal situation, appropriately applied HIT can reduce clinicians' workload and reverse the increased documentation burden to make physicians' work more patient-centered again with more time allowed for face-to-face interaction, increasing both patient and staff satisfaction. This is more the case in LMICs where these modalities could offer the opportunity to leapfrog in achieving Universal Health Coverage.

Despite all the benefits and advantages that these technologies deliver, one must also consider potential problems that halt, or arise from, their integration: Healthcare systems are extremely rigid to change, many stakeholders have very disparate or misaligned incentives, there is resilience towards change and reluctance by users, there is a global increase in the demand of care leading to rising costs, all of which 
is paired with persisting difficulties of data fragmentation and lacking HIT interoperability. These factors display significant barriers that must be overcome in order to drive change in the right direction. As the challenges are multifactorial, multifaceted teams are required to tackle them. This requires a culture change to overhaul long-established operational processes and workflows, which are highly complex and involve a host of actors, in order to work towards optimal digitalization. Newly arising problems, such as interoperability issues between varied health information systems established in isolation, continue to be a substantial challenge for a smooth information exchange. On top of this, the integration of new technologies to solve old problems paves the way for new problems to arise. These revolve not only around financial implications (e.g., maintenance costs) but also around regulatory and ethical issues. As with IDx-DR, the U.S. Department of Health and Human Services for Food and Drug Administration (FDA) has approved their algorithm as medical device and granted its unsupervised use (Van Der Heijden et al. 2018). However, AI algorithms function differently to what was traditionally defined as medical device, because they must constantly evolve to maintain their accuracy. This high amount of uncertainty demands great attention for a regulatory and ethical debate going forward. In the global context, this might give rise to an increase in health disparity between the have and the have-not.

The complexity of challenges that health systems are faced with increases. Despite data science bringing hope to solve many long-established problems in the face of aforementioned difficulties in the smooth collection, exchange, and subsequent analysis of data, bringing change from bench to bedside cannot be done by technology alone and involves mainly human factors. The rise of algorithms to enhance medical diagnosis and decision-making are only of value when they are being implemented in day-to-day practice and accessible to the entire global community. In this regard, the efforts of organizations, such as Sana (MIT), are of paramount importance in promoting data science in global health (Angelidis et al. 2016).

\subsection{Conclusion}

In order to universally improve healthcare, countries need to balance cost, quality, and access, and HIT has proven to have the potential in addressing these needs. Using it and applying principles of data science allows information to be stored, processed, exchanged, and analyzed in order to maximize its value and use. If used effectively, it can lead to a more efficient and safe delivery of care, improved access to information and services for an evidence-based decision-making, and enhance both clinicians' and patients' satisfaction. However, the necessary HIT requires a conducive ICT-enabled environment in order to facilitate the greatest possible degree of interoperability and cross-disciplinary collaboration.

All technological advancements and their possibilities are of limited use, however, when they cannot be implemented and utilized effectively due to a misalignment with the human layer. For example, when designing HIT applications, the user experience 
must be equally important as its anticipated improvement in clinical effectiveness and efficiency. Likewise must stakeholders communicate through agreed terminology and work towards a common goal. This can only be achieved by an evenly close collaboration of software developers with future users, governments with healthcare managers, and data scientists with clinicians. Especially in the context of LMICs, joint forces between professions and regions are likely to have better outcomes than many individual efforts to innovate healthcare. Here, governmental drive can be an important initiating force in fostering the necessary interprofessional partnerships, which should ideally go beyond organizational or regional interests.

The utilization of data science has the potential to tackle some of the greatest challenges in modern healthcare that exist on various different levels, eventually contributing to making advancements in global health. Although, in order for HIT applications to function as they have been designed currently and will be designed in the future, all stakeholders need to collaborate in tackling technical, behavioural, financial, ethical, and regulatory challenges to enable the best possible use of these technologies for leveraging data science in global health.

\section{References}

AIMed, B. (2018). The ethical imperative of learning from the data-AI Med [WWW document]. AI Med. Retrieved October 22, 2018, from http://ai-med.io/the-ethical-imperative-of-learningfrom-the-data/.

Akhlaq, A., McKinstry, B., Muhammad, K. B., \& Sheikh, A. (2016). Barriers and facilitators to health information exchange in low- and middle-income country settings: A systematic review. Health Policy and Planning, 31, 1310-1325.

Al-Shorbaji, N. (2018). The world health assembly resolutions on eHealth: eHealth in support of universal health coverage. Methods of Information in Medicine, 52, 463-466.

Angelidis, P., Berman, L., Casas-Perez, M. de la, L., Celi, L. A., Dafoulas, G. E., Dagan, A., Escobar, B., Lopez, D. M., Noguez, J., Osorio-Valencia, J. S., Otine, C., Paik, K., Rojas-Potosi, L., Symeonidis, A. L., Winkler, E. (2016). The hackathon model to spur innovation around global mHealth. Journal of Medical Engineering \& Technology, 40, 392-399.

Ash, J. S., Sittig, D. F., Campbell, E. M., Guappone, K. P., Dykstra, R. H. (2007). Some unintended consequences of clinical decision support systems. AMIA Annual Symposium Proceedings, 26-30.

Backman, R., Bayliss, S., Moore, D., \& Litchfield, I. (2017). Clinical reminder alert fatigue in healthcare: A systematic literature review protocol using qualitative evidence. Systematic Review, 6, 255.

Baller, S., Dutta, S., \& Lanvin, B. (2016). The global information technology report 2016. World Economic Forum.

Blijleven, V., Koelemeijer, K., Wetzels, M., \& Jaspers, M. (2017). Workarounds emerging from electronic health record system usage: Consequences for patient safety, effectiveness of care, and efficiency of care. JMIR Hum Factors, 4, e27.

Celi, L. A. (2019). Global health informatics to improve quality of care [WWW document]. edX. Retrieved April 17, 2019 https://www.edx.org/course/global-health-informatics-to-improve-qua lity-of-care.

Clifford, G. D. (2016). E-health in low to middle income countries. Journal of Medical Engineering \& Technology, 40, 336-341. 
Coiera, E. W. (1996). Artificial intelligence in medicine: The challenges ahead. Journal of the American Medical Informatics Association, 3, 363-366.

Costa, C. M., Gondim, D. D., Gondim, D. D., Soares, H. B., Ribeiro, A. G. C. D., Silva, I., et al. (2012). S2DIA: A diagnostic system for diabetes mellitus using SANA platform. Conference on Proceedings of IEEE Engineering in Medicine and Biology Society, 2012, 6078-6081.

Dankwa-Mullan, I., Rivo, M., Sepulveda, M., Park, Y., Snowdon, J., \& Rhee, K. (2018). Transforming diabetes care through artificial intelligence: The future is here. Population Health Management.

Eden, K. B., Totten, A. M., Kassakian, S. Z., Gorman, P. N., McDonagh, M. S., Devine, B., et al. (2016). Barriers and facilitators to exchanging health information: A systematic review. International Journal of Medical Informatics, 88, 44-51.

Esteva, A., Kuprel, B., Novoa, R. A., Ko, J., Swetter, S. M., Blau, H. M., et al. (2017). Dermatologistlevel classification of skin cancer with deep neural networks. Nature, 542, 115-118.

FDA. (2018). FDA news release-FDA permits marketing of artificial intelligence-based device to detect certain diabetes-related eye problems [WWW document]. U.S. Food and Drug Administration. Retrieved April 4, 2019, from https://www.fda.gov/newsevents/newsroom/pressannounc ements/ucm604357.htm.

Finn, N. (2011). Health information exchange: a stepping stone toward continuity of care and participatory medicine. Med: J. Particip.

Garbuio, M., \& Lin, N. (2019). Artificial intelligence as a growth engine for health care startups: Emerging business models. California Management Review, 61, 59-83.

Goldberg, D. J. (2018). The burden of electronic health record implementation [WWW document]. Dermatology Times. Retrieved November 11, 2018, from http://www.dermatologytimes.com/ legal-eagle/will-dr-emr-have-more-or-less-liability-his-new-electronic-health-records.

Gordon, W. J., Catalini, C. (2018). Blockchain technology for healthcare: Facilitating the transition to patient-driven interoperability [WWW document]. Retrieved October 22, 2018, from https:// www.ncbi.nlm.nih.gov/pubmed/30069284.

Gordon, W., Wright, A., Landman, A. (2017). Blockchain in health care: Decoding the hype [WWW document]. NEJM catalyst. Retrieved October 22, 2018, from https://catalyst.nejm.org/decodingblockchain-technology-health/.

HealthIT.gov. (2017). Health information exchange [WWW document]. HealthIT.gov-Health IT and health information exchange basics: health information exchange. Retrieved November 18, 2018, from https://www.healthit.gov/topic/health-it-basics/health-information-exchange.

Khairat, S., Marc, D., Crosby, W., \& Al Sanousi, A. (2018). Reasons For Physicians Not Adopting Clinical Decision Support Systems: Critical Analysis. JMIR Med Inform, 6, e24.

Komorowski, M., Celi, L. A., Badawi, O., Gordon, A. C., \& Faisal, A. A. (2018). The artificial intelligence clinician learns optimal treatment strategies for sepsis in intensive care. Nature Medicine, 24, 1716-1720.

Koplan, J. P., Bond, T. C., Merson, M. H., Reddy, K. S., Rodriguez, M. H., Sewankambo, N. K., Wasserheit, J. N. (2009). Consortium of universities for global health executive board. Towards a common definition of global health. Lancet, 373, 1993-1995.

Mangalmurti, S. S., Murtagh, L., \& Mello, M. M. (2010). Medical malpractice liability in the age of electronic health records. New England Journal of Medicine, 363, 2060-2067.

Metcalf, D. (2019). Blockchain in healthcare: Innovations that empower patients, connect professionals and improve care. Taylor \& Francis.

Mills, P. R., Weidmann, A. E., \& Stewart, D. (2017). Hospital staff views of prescribing and discharge communication before and after electronic prescribing system implementation. International Journal of Clinical Pharmacy, 39, 1320-1330.

Murphy, E. V. (2014). Clinical decision support: Effectiveness in improving quality processes and clinical outcomes and factors that may influence success. Yale Journal of Biology and Medicine, $87,187-197$.

Ng, A. (2011). Machine learning [WWW Document]. Coursera. Retrieved 17 April, 2019, from https://www.coursera.org/learn/machine-learning. 
Obermeyer, Z., \& Emanuel, E. J. (2016). Predicting the future-Big data, machine learning, and clinical medicine. New England Journal of Medicine, 375, 1216-1219.

Puaar, S. J., \& Franklin, B. D. (2017). Impact of an inpatient electronic prescribing system on prescribing error causation: A qualitative evaluation in an English hospital. BMJ Quality Safety, 27, 529-538.

Sadoughi, F., Nasiri, S., \& Ahmadi, H. (2018). The impact of health information exchange on healthcare quality and cost-effectiveness: A systematic literature review. Computer Methods and Programs in Biomedicine, 161, 209-232.

Shanafelt, T. D., Dyrbye, L. N., Sinsky, C., Hasan, O., Satele, D., Sloan, J., et al. (2016). Relationship between clerical burden and characteristics of the electronic environment with physician burnout and professional satisfaction. Mayo Clinic Proceedings, 91, 836-848.

Silow-Carroll, S., Edwards, J. N., \& Rodin, D. (2012). Using electronic health records to improve quality and efficiency: The experiences of leading hospitals. Issue Brief, 17, 1-40.

Singh, H., \& Sittig, D. F. (2016). Measuring and improving patient safety through health information technology: The health IT safety framework. BMJ Quality \& Safety, 25, 226-232.

Sundin, P., Callan, J., \& Mehta, K. (2016). Why do entrepreneurial mHealth ventures in the developing world fail to scale? Journal of Medical Engineering \& Technology, 40, 444-457.

The Alan Turing Institute. (2018). The Alan turing institute. Retrieved October 22, 2018, from https://www.turing.ac.uk.

Van Der Heijden, A. A., Abramoff, M. D., Verbraak, F., van Hecke, M. V., Liem, A., \& Nijpels, G. (2018). Validation of automated screening for referable diabetic retinopathy with the IDx-DR device in the Hoorn diabetes care system. Acta Ophthalmologica, 96, 63-68.

Washington, V., DeSalvo, K., Mostashari, F., \& Blumenthal, D. (2017). The HITECH era and the path forward. New England Journal of Medicine, 377, 904-906.

Weingart, S. N., Simchowitz, B., Padolsky, H., Isaac, T., Seger, A. C., Massagli, M., et al. (2009). An empirical model to estimate the potential impact of medication safety alerts on patient safety, health care utilization, and cost in ambulatory care. Archives of Internal Medicine, 169, 14651473.

World Health Assembly. (2005). WHA58.28 eHealth.

World Health Organization. (2013). The world health report 2013: Research for universal health coverage. World Health Organization.

World Health Organization. (2016). Atlas of eHealth country profiles 2015: The use of eHealth in support of universal health coverage Based on the findings of the 2015 global survey on eHealth. World Health Organization.

Yu, K.-H., Kohane, I. S. (2018). Framing the challenges of artificial intelligence in medicine. BMJ Quality \& Safety bmjqs 2018-008551.

Open Access This chapter is licensed under the terms of the Creative Commons Attribution 4.0 International License (http://creativecommons.org/licenses/by/4.0/), which permits use, sharing, adaptation, distribution and reproduction in any medium or format, as long as you give appropriate credit to the original author(s) and the source, provide a link to the Creative Commons license and indicate if changes were made.

The images or other third party material in this chapter are included in the chapter's Creative Commons license, unless indicated otherwise in a credit line to the material. If material is not included in the chapter's Creative Commons license and your intended use is not permitted by statutory regulation or exceeds the permitted use, you will need to obtain permission directly from the copyright holder.

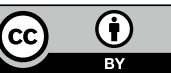

